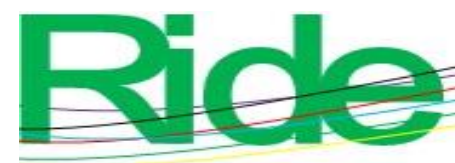

Revista Iberoamericana para la Investigación y el Desarrollo Educativo ISSN 2007 - 7467

https://doi.org/10.23913/ride.v11i21.806

Artículos científicos

\title{
Análisis socioeducativo de la empatía por los números aplicado a 228 alumnos de la carrera de psicología del Centro Universitario de la Costa U. de G.
}

\begin{abstract}
Socio-educational analysis of empathy for numbers applied to 228 students of the psychology career of the Centro Universitario de la Costa U. de G.
\end{abstract}

\section{Análise socioeducativa de empatia por números aplicada a 228 alunos de psicologia do Centro Universitário da Costa U. de G.}

Claudio Rafael Vásquez Martínez

Universidad de Guadalajara, México

crvasquezm@gmail.com

https://orcid.org/0000-0001-6383-270X

Felipe Anastacio González González Universidad Autónoma de Tamaulipas, México fgonzale28@hotmail.com https://orcid.org/0000-0002-1410-8616

\section{Resumen}

El objeto del presente trabajo fue analizar la empatía por los números de estudiantes de la carrera de Psicología del Centro Universitario de la Costa, Universidad de Guadalajara. La muestra estuvo constituida por 228 jóvenes escolarizados, de edades comprendidas entre los 21 y los 24 años, de los cuales 115 eran hombres y 113 mujeres. Los resultados demuestran que los alumnos no sienten empatía por los números debido a que no los entienden, son abstractos y se frustran al no saber realizar las operaciones. Esto ocasiona que abandonen la iniciativa de aprender matemáticas. Se concluye, por tanto, que si no se cambia esta percepción de frustración y miedo al realizar operaciones numéricas, los futuros egresados podrían desaprovechar los beneficios que ese conocimiento ofrece en la vida cotidiana. 

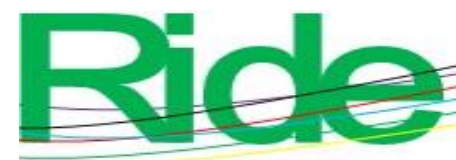

Revista Iberoamericana para la Investigación y el Desarrollo Educativo

ISSN 2007 - 7467

Palabras claves: aprendizaje, empatía por los números, socioeducativo.

\section{Abstract}

The purpose of this work was to analyze the empathy for the numbers of students of the Psychology career of the University Center of the Coast, University of Guadalajara. The sample consisted of 228 young people in school, aged between 21 and 24 years, of which 115 were men and 113 women. The results show that the students do not feel empathy for the numbers because they do not understand them, they are abstract and they get frustrated by not knowing how to perform the operations. This causes them to give up the initiative to learn math. It is concluded, therefore, that if this perception of frustration and fear is not changed when performing numerical operations, future graduates could miss the benefits that this knowledge offers in daily life.

Keywords: learning, empathy for numbers, socioeducational.

\section{Resumo}

O objetivo do presente trabalho foi analisar a empatia pelos números de alunos da carreira de Psicologia do Centro Universitário do Litoral da Universidade de Guadalajara. A amostra foi composta por 228 jovens em idade escolar, com idades entre 21 e 24 anos, sendo 115 homens e 113 mulheres. Os resultados mostram que os alunos não sentem empatia pelos números porque não os compreendem, são abstratos e ficam frustrados quando não sabem realizar as operações. Isso os faz desistir da iniciativa de aprender matemática. Conclui-se, portanto, que se essa percepção de frustração e medo não for alterada ao realizar operações numéricas, os futuros graduados podem perder os benefícios que esse conhecimento oferece no cotidiano.

Palavras-chave: aprendizagem, empatia por números, socioeducativa.

Fecha Recepción: Junio 2020

Fecha Aceptación: Diciembre 2020 


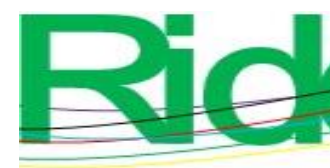

Revista lberoamericana para la Investigación y el Desarrollo Educativo ISSN 2007 - 7467

\section{Introducción}

El razonamiento matemático es una práctica que cualquier estudiante universitario debe desarrollar. Por ejemplo, un investigador del campo de la psicología — especialidad en donde se enfoca este trabajo - necesita de la estadística para organizar los datos recabados de sus grupos de estudio (Mendenhall, Beaver y Beaver, 2017). El problema, sin embargo, radica en que algunas veces el alumno asume una posición de rechazo ante este tipo de conocimiento, lo cual dificulta el proceso de enseñanza y aprendizaje. En palabras de AlonsoCanovas, Fernández-Estévez y Sánchez-Santad (2008), el cerebro necesita adoptar una actitud activa para comprender un razonamiento, por lo que se puede asumir que si el estudiante no está dispuesto a conocer la manera en que se puede resolver un problema matemático, no podrá entender los principios deductivos que se requieren para hallar la respuesta buscada.

Paenza (2006) explica que el problema con las matemáticas no es exclusivo de un país o de una única generación, sino que es una dificultad experimentada en todo el mundo y en cualquier época, a pesar de que ese conocimiento es esencial para que las personas fomenten su creatividad.

Por ese motivo, el objeto del presente trabajo fue analizar la empatía por los números de estudiantes de la carrera de psicología del Centro Universitario de la Costa, Universidad de Guadalajara.

\section{Hipótesis}

1. Los estudiantes de la carrera de psicología del Centro de la Universidad de la Costa (U. de G.) no muestran empatía por los números debido a que no saben cómo realizar los procesos matemáticos para llevar a cabo las operaciones con éxito.

2. No hay empatía por los números debido a que exigen razonamientos abstractos que no comprenden.

\section{Marco teórico}

Los números son entes que se hallan omnipresentes en nuestro diario vivir, bien sea cuando se paga una deuda, cuando se recibe un depósito bancario, cuando se calcula el tiempo para trasladarse a un lugar, etc. Todos esos procedimientos numéricos de organización, análisis e interpretación se sustentan en los principios de una ciencia llamada estadística 


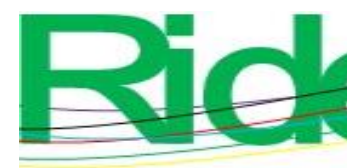

\section{Revista Iberoamericana para la Investigación y el Desarrollo Educativo ISSN 2007 - 7467}

(Lipschutz y Schiller, 2000). Sin embargo, la experiencia demuestra que muchos estudiantes no sienten empatía ${ }^{1}$ por aprender a resolver problemas de esa índole.

Según Alonso-Canovas et al. (2008), un porcentaje de esa responsabilidad recae en los propios estudiantes cuando no están dispuestos a esforzarse por comprender los principios esenciales del razonamiento matemático. En otras palabras, el alumno debe ser capaz de activar sus estrategias resolutivas para resolver un problema.

Sin embargo, para Paenza (2006) el peor enemigo de las matemáticas es el docente, ya que no logra despertar en los jóvenes la curiosidad mínima para poder disfrutarla. "La matemática, tal como se enseña, no seduce a nadie. Es como si nos obligaran a querer lo que no queremos. Y por eso la rebelión de los jóvenes que se resisten y la rechazan” (Paenza, 2008, p. 168). Por eso, Alonso-Canovas et al. (2008, p. 7) ofrecen algunas sugerencias al momento de enseñar las matemáticas:

1. Las matemáticas deben de ser motivadoras: Los estudiantes no encuentran relación entre lo que aprenden en el aula y su entorno porque los conceptos explicados se suelen usar en situaciones hipotéticas; es decir, no se emplean para solucionar un problema cotidiano, como los que surgen en partidos de fútbol, videojuegos o cualquier actividad más familiar para ellos.

2. El aprendizaje debe ser demostrativo, basado en razonar y deducir: Los estudiantes memorizan fórmulas y ejemplos, pero posteriormente se les cambia el problema, de modo que no sabrán cómo resolverlo debido a que aún no han desarrollado su agilidad matemática.

3. El problema debe ser planteado: En clase no solo se debe buscar la respuesta, sino también la pregunta y los datos necesarios para resolverla; solo así se crearán problemas reales que contribuirán al desarrollo de la creatividad del estudiante.

4. El docente debe tener en cuenta que las matemáticas son de carácter acumulativo: Solo se puede comprender una nueva explicación si se han asimilado bien los conceptos previos que la sustentan. En caso contrario, la experiencia con los números será desagradable, por lo que el estudiante terminará rechazando a las matemáticas (Rius, 21 de mayo de 2015).

\footnotetext{
1 El término empatía, de forma general, se usa como sinónimo de 'agrado', e implica un cierto placer, familiaridad y satisfacción por la actividad desarrollada. Por su parte, el vocablo número se refiere a la base de todo sistema numérico, el cual se representa por medio de signos numéricos (Deconceptos, 2009).
} 


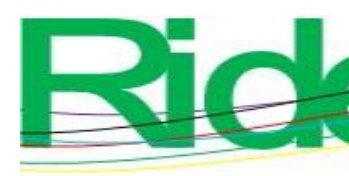

\section{Revista Iberoamericana para la Investigación y el Desarrollo Educativo ISSN 2007 - 7467}

Aunado a lo anterior, vale destacar que los docentes deben demostrar la utilidad que subyace en el razonamiento matemático, pues como explica Musk (2016) "nuestro cerebro ha evolucionado para desechar la información que piensa es irrelevante" (p. 5). Para este autor, el aprendizaje debe estar enfocado en resolver un problema específico mediante el uso de una herramienta que se debe dominar. Al respecto, se debe tener en cuenta que las personas, a veces de manera inconsciente, se suelen plantear este tipo de preguntas cuando se enfrentan a un nuevo conocimiento: “¿Por qué estoy aprendiendo esto? o ¿Por qué se me pide que resuelva estos problemas tan extraños?”.

Por eso, Paenza (2016) ofrece algunas ideas sobre las actividades que desmotivan a los estudiantes.

- El exponente o docente brinda a los estudiantes respuestas a preguntas que no tienen, por lo cual el tema no les interesa.

- En el aula se trabajan problemas que no tienen ninguna aplicación práctica en la vida actual, por lo cual los estudiantes no encuentran utilidad en lo que se les enseña.

- Introducir el tema de las matemáticas del modo menos atractivo, es decir, se le presenta a los estudiantes como algo tedioso y laborioso, en lugar de algo práctico y creativo mediante la resolución de juegos o acertijos.

- El recuerdo de la humillación es uno de los principales motivos por lo que la gente tiene un trauma con las matemáticas, recuerdos que traen una sensación de frustración que vinculan a las matemáticas.

- La duda de la recompensa o el beneficio de saber aplicar los números, es decir, los estudiantes no toleran la frustración hacia las matemáticas porque no observan el provecho de ese conocimiento. En otras palabras, se debe pensar en el proceso seguido para aprender a conducir, donde el aprendiz se esfuerza porque es consciente de que al final logrará manejar un automóvil y pasear en él.

- El creer que en las matemáticas todo está escrito y descubierto; es decir, las matemáticas se tienen que asumir como las ciencias de la salud, donde aún faltan operaciones y planteamientos por resolver.

- El conocimiento se debe socializar, lo que implica dejar de castigar a la persona que no sabe. En otras palabras, el hecho de ser un padre o maestro no significa que se tengan todas las respuestas. De hecho, las respuestas a los problemas se deben buscar de manera conjunta (Paenza, 2016). 


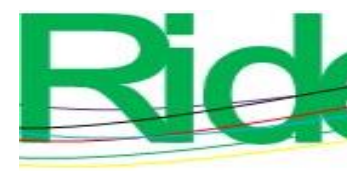

Revista Iberoamericana para la Investigación y el Desarrollo Educativo

ISSN 2007 - 7467

Otro factor que entorpece al aprendizaje de los números es la velocidad, la cual tiene efectos negativos en el aprendizaje. En otras palabras, la memoria falla cuando las personas están estresadas o presionadas con el tiempo a la hora de aprender. Sumado a esto, se debe procurar trabajar más para desarrollar las habilidades que para conseguir buenas calificaciones. Por ello, Boaler (2015) concluye que lo que necesitan las matemáticas es devolver una visión abierta y creativa a los estudiantes, así como alentar la creatividad y el razonamiento de estos (KienyKe, 2019). De lo contrario, de las escuelas seguirán egresando personas con un profundo rechazo y desconocimiento de ese saber básico (Soto, 15 de febrero de 2019).

Un ejemplo de ello es la prueba realizada por la Secretaría de Educación Pública, en la cual se demuestra que $64.5 \%$ de los alumnos de secundaria son capaces de hacer operaciones simples (como restas, sumas o multiplicaciones), mientras que solo $21.7 \%$ puede hacer estas operaciones con decimales. También se ha documentado que únicamente $8.6 \%$ de los alumnos de secundaria pueden realizar operaciones como fracciones, potencias y expresiones algebraicas. Por lo anterior, para Soto (15 de febrero de 2019) es importante promover el conocimiento financiero, ya que promueve en las personas la adquisición de habilidades para mejorar sus vidas y acceder a mejores oportunidades.

En este contexto, no se puede evitar pensar con nostalgia en el pasado y preguntar cuáles fueron los factores que impulsaron esta situación. Se debe pensar que hace muchos años la cultura mexicana realizó grandes obras de ingeniería, aportaciones a la física y a las matemáticas, como el calendario maya y azteca, la consideración del cero como número o la construcción de las pirámides (Torres, s. f.).

Es urgente, por tanto, trabajar para cambiar el actual escenario. Para ello, se debe tomar como ejemplo a nuestros ancestros y convertir a los números en entidades con las cuales se puede aprender, incluso de manera lúdica.

\section{Marco metodológico \\ Participantes}

De los 562 alumnos del ciclo escolar 2019 A de la carrera de licenciado en Psicología del Centro Universitario de la Costa de la U. de G., se eligió una muestra de 228 estudiantes, para lo cual se aplicó el sistema de muestreo probabilístico, específicamente el muestreo 

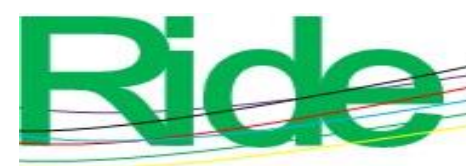

Revista Iberoamericana para la Investigación y el Desarrollo Educativo ISSN 2007 - 7467

aleatorio simple, en el cual — según Salkind (1999) — cada elemento de la población tiene una probabilidad igual e independiente de ser seleccionado.

\section{Muestra}

Para el desarrollo de la presente investigación científica la muestra fue de 228 jóvenes escolarizados, de edades comprendidas entre los 21 y los 24 años, de los cuales 115 eran hombres y 113 mujeres. Además del sexo y la edad de cada sujeto de la investigación, Hernández, Fernández y Baptista (2014) mencionan que las características de la población pueden clasificarse atendiendo a tres aspectos: 1) de contenido: estas características se refieren a que los sujetos u objetos a investigar posean la información requerida para ello; 2) de lugar: se refiere a especificar claramente los límites geográficos y el tipo de institución en donde se realizará la investigación, y 3) de tiempo: esta característica se refiere al periodo en que los sujetos cumplen con las otras características.

En este caso en particular, la población correspondió a la matrícula de los estudiantes del ciclo escolar 2019 A (enero a mayo) de la carrera de licenciado en Psicología del Centro Universitario de la Costa de la U. de G. La muestra es la "parte o fracción representativa de un conjunto de una población, universo o colectivo, que ha sido obtenida con el fin de investigar ciertas características del mismo” (Ander-Egg, 1995, p. 179).

Para obtener la muestra de sujetos efectivos, se colocaron pequeños trozos de papel doblados con números del 1 al 562 (matrícula de alumnos), los cuales luego se colocaron en una tómbola en donde se realizó el tratamiento estadístico. Después, se procedió al análisis de los datos en la sala de maestros del Centro Universitario de la Costa de la U. de G. a las 9:00 de la mañana del 2 de mayo del año 2019.

La probabilidad fue igual, ya que no existió predisposición para elegir a un elemento o a otro; y fue independiente porque la selección de un elemento no influyó para elegir a otro. Para determinar el tamaño de la muestra se aplicó la siguiente fórmula:

$$
\mathrm{n}=\frac{Z^{2} P q N}{\mathrm{~N} e^{2}+Z^{2} P q}
$$

$\mathrm{n}=$ Tamaño de la muestra.

$\mathrm{Z}=$ Nivel de confianza deseado.

$\mathrm{P}=$ Proporción de la población con la característica deseada (éxito). 
$\mathrm{q}$ = Proporción de la población sin la característica deseada (fracaso).

$\mathrm{e}=$ Nivel de error dispuesto a cometer.

$\mathrm{N}=$ Tamaño de la población.

En donde:

$Z=$ Nivel de confianza deseado $95 \%$

$\mathrm{P}=\mathrm{El}$ éxito deseado $50 \%$

$\mathrm{q}=$ Fracaso deseado $50 \%$

$\mathrm{e}=$ Nivel de error $5 \%$

$\mathrm{N}=$ Tamaño de la población de 562 (matrícula).

Desarrollo: $\mathrm{n}=\mathrm{X}, \mathrm{Z}=1.96$

$P=0.5 q=0.5$

$\mathrm{E}=\mathbf{0 . 0 5} \mathrm{N}=\mathbf{5 6 2}$

$$
\begin{aligned}
& n=\frac{(1.96)^{2}}{562 *} \frac{* 0.5 * 0.5 * 562}{(0.05)^{2}+(1.96)^{2} * 0.5 * 0.5} \\
& \boldsymbol{n}=\frac{\mathbf{5 3 9 . 7 4}}{\mathbf{2 . 3 6 5 4}}
\end{aligned}
$$

$\boldsymbol{n}=\mathbf{2 2 8}$

El tamaño determinado de la muestra fue de 228.

\section{Análisis}

En la figura 1 se puede observar que el uso de los números en la vida cotidiana del estudiante es muy frecuente; esto significa que es importante promover el gusto por los números. 
Figura 1. Uso de los números
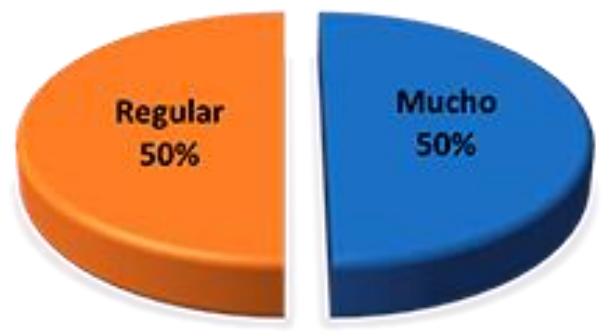

Fuente: Elaboración propia

En la figura 2 se puede apreciar que la mitad de los alumnos sienten gusto por las matemáticas, aspecto que se debe tomar en cuenta en la intención de implantar el gusto por los números.

Figura 2. Empatía por las matematicas
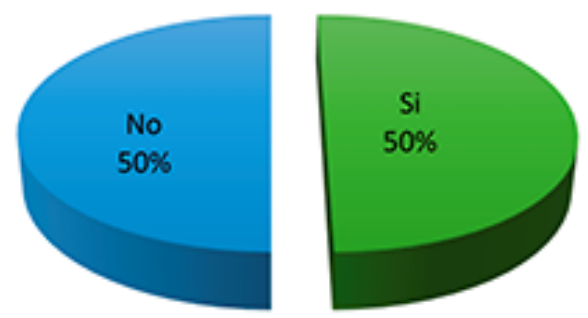

Fuente: Elaboración propia

En la figura 3 se observa que los estudiantes están convencidos de que la estadística es vital para el funcionamiento diario, de ahí que sea importante aprenderla. 
Figura 3. Utilidad de aprender estadística

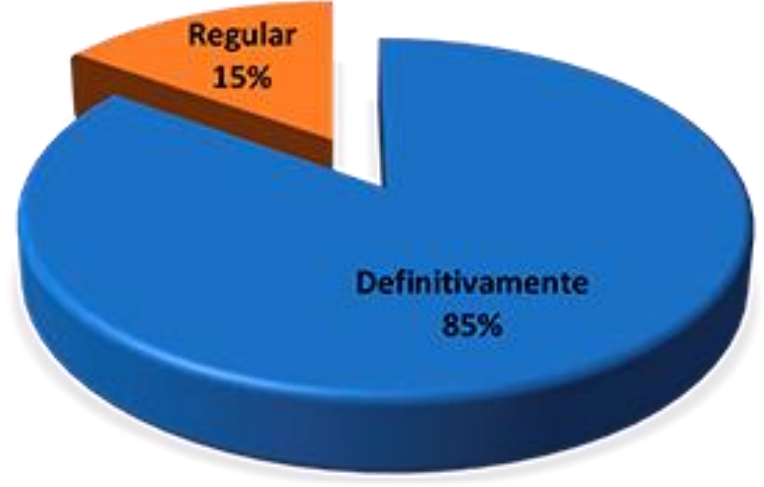

Fuente: Elaboración propia

En la figura 4 se observa que los alumnos presentan sentimientos tanto optimistas como pesimistas, son conscientes de la importancia de aprender a realizar operaciones e incluso les gustan las operaciones matemáticas, la mayoría se sienten nerviosos e inseguros al realizarlas.

Figura 4. Sentimiento al realizar operaciones

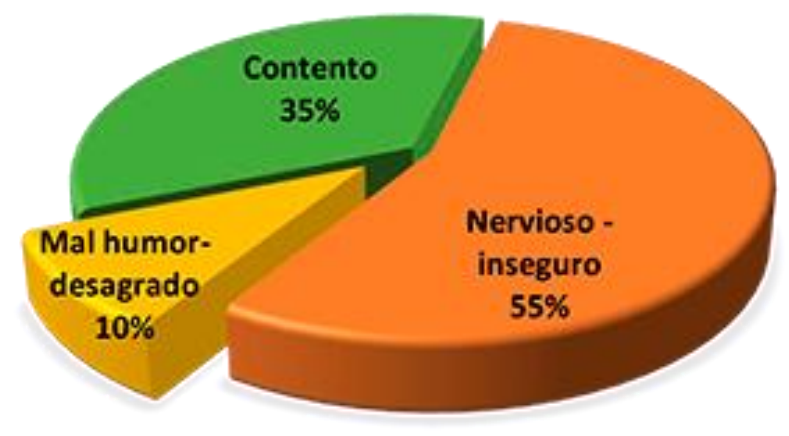

Fuente: Elaboración propia

En la figura 5 se evidencia que la mitad de los alumnos están dispuestos a repetir los ejercicios para conseguir la respuesta correcta, es decir, se puede utilizar esa determinación para lograr cambios significativos en el aprendizaje de los números. 
Figura 5. Reacción al no poder realizar una operación
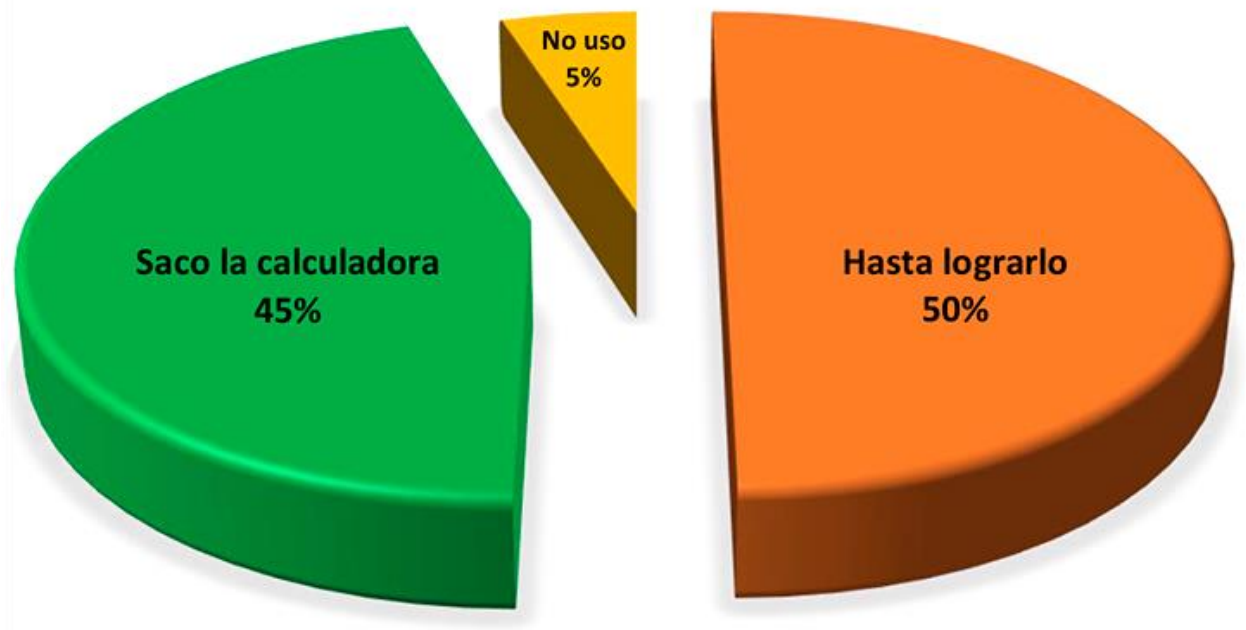

Fuente: Elaboración propia

En figura 6 los estudiantes ofrecen sugerencias sobre cómo les gustaría aprender a realizar operaciones en el salón de clase. Del total, $40 \%$ siente interés al realizar operaciones reales que puedan entender porque las viven en situaciones cotidianas, mientras que $45 \%$ menciona que les gustaría aprender cómo realizar operaciones con ejercicios hechos en el salón de clase y solo el 5\% no lo intentó.

Figura 6. Sugerencias para el aprendizaje de los números

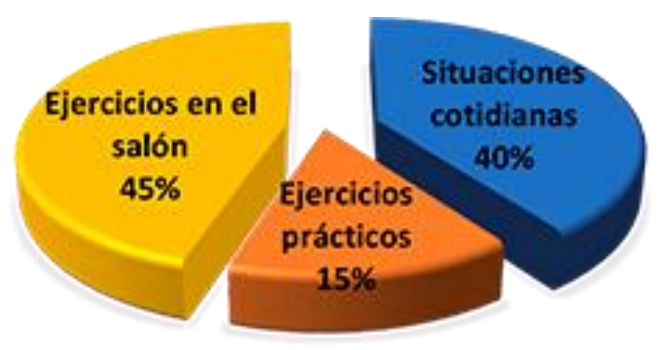

Fuente: Elaboración propia 



Revista Iberoamericana para la Investigación y el Desarrollo Educativo ISSN 2007 - 7467

\section{Discusión}

Al interpretar y evaluar los resultados obtenidos, se halló que los alumnos tienden a limitar su motivación hacia la comprensión y uso de los números por la falta de una metodología oportuna de acuerdo con el contexto del nivel universitario. Asimismo, los resultados indican que los alumnos de la muestra prefieren las actividades prácticas para poder asimilar lo abstracto de los números.

Si bien se sabe que los procesos matemáticos son importantes para el diario vivir, es necesario fortalecer dicha idea, ya que los jóvenes aspirantes a un nivel universitario podrían creer que estudiando determinada carrera se podrán librar del esfuerzo que implica el desarrollo del razonamiento matemático.

\section{Conclusión}

Al analizar lo expuesto anteriormente, se puede confirmar la hipótesis planteada, es decir, los estudiantes de la carrera de Psicología del Centro Universitario de la Costa U. de G. no sienten empatía por los números debido sentimientos de nerviosismo ya que no los entienden, son abstractos y se frustran al no saber realizar las operaciones. Esto ocasiona que abandonen la iniciativa de aprender matemáticas.

También se concluye que si no se cambia esta percepción de frustración y miedo al realizar operaciones con números, los futuros egresados podrían desaprovechar los beneficios que ese conocimiento ofrece en la vida cotidiana por ejemplo al manejar sus finanzas.

Como conclusión general se debe insistir en la importancia de cambiar las estrategias usadas en el aprendizaje de las matemáticas, pues de ese modo los alumnos podrán sentir más motivación hacia este tipo de contenido.

\section{Recomendaciones}

Luego de analizar la información recopilada se pueden ofrecer algunas recomendaciones:

1. El aprendizaje de los números u operaciones se debe realizar con base en algún tema que sea de interés para los estudiantes.

2. Los ejercicios deben ser aplicables a la vida cotidiana, por lo que deben ser prácticos y específicos. 


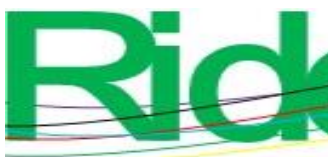

Revista Iberoamericana para la Investigación y el Desarrollo Educativo

ISSN 2007 - 7467

3. Los estudiantes no deben dar solo las respuestas, sino que también deben plantear el problema.

4. El docente o quien imparte la enseñanza debe dejar claro un concepto antes de pasar al siguiente.

5. El aprendizaje matemático debe ser multisensorial, es decir, debe incluir a la vista, al tacto y al oído.

\section{Futuras líneas de investigación}

De este estudio se desprenden futuras líneas de investigación:

- Estudios en aulas de clase en primaria, secundaria, preparatoria y universidad sobre empatía por los números.

- Estudios locales en centros de estudios educativos sobre los efectos de las pandemias y post pandemias con sus casos de resiliencia en la aplicación de la empatía por los números.

\section{Referencias}

Alonso-Canovas, D., Fernández-Estévez, M. A. y Sánchez-Santad, F. (2008). El cerebro musical. Almería: Universidad de Almería.

Ander-Egg, E. (1995). Técnicas de investigación social. Buenos Aires: Lumen.

DeConceptos. (2009). Concepto de número. Recuperado de https://deconceptos.com/matematica/numero

Hernández, R., Fernández, C. y Baptista, P. (2014). Metodología de la investigación. México D. F.: Editorial McGraw-Hill.

KienyKe (2017). ¿No le gustan las matemáticas? Esta sería la razón. Recuperado de https://www.kienyke.com/historias/no-le-gustan-las-matematicas-esta-seria-la-razon

Lipschutz, S. y Schiller, J. (2000). Introducción a la probabilidad y estadística. España: Mc Graw Hill.

Management (20 de julio de 2017). ¿Por qué no te gustan las matemáticas? El fundador de Tesla tiene la respuesta. Recuperado de https://www.dineroenimagen.com/2017-0720/88981

Mendenhall, W., Beaver, R. y Beaver, B. (2017). Probabilidad y estadística para las ciencias sociales del comportamiento y la salud. México: CENGAGE learning. 


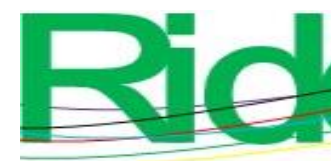

Revista lberoamericana para la Investigación y el Desarrollo Educativo

ISSN 2007 - 7467

Muks, E. (2016). El empresario que anticipa el futuro. Ciudad de México: Ediciones Península.

Paenza, A. (2006). Matemáticas, ¿estás ahí? Sobre números, personajes, problemas y curiosidades. Buenos Aires: Siglo veintiuno.

Paenza, A. (2008). Matemática ... ¿estás ahi? Episodio 100. Buenos Aires: Siglo XXI.

Paenza, A. (2016). 7 motivos por los que a muchos no le gustan las matemáticas. Diario Veloz. Recuperado de http://www.diarioveloz.com/notas/169728-7-motivos-los-quemuchos-no-le-gustan-las-matematicas

Rius, M. (21 de mayo de 2015). ¿Por qué muchos estudiantes odian las matemáticas? Recuperado de https://www.lavanguardia.com/vida/20150521/54431772174/estudiantes-odianmatematicas.html/

Salkind, N. (1999). Método de investigación. México, D.F.: Prentice-Hall.

Soto, S. (15 de febrero de 2019). No saber matemáticas condena a los mexicanos a la pobreza. Recuperado de https://www.dineroenimagen.com/no-saber-matematicasmexicanos-pobreza

Torres, S. (s. f.) Breve historia de las matemáticas en México. Departamento de Matemáticas Facultad de Ciencias UNAM. Recuperado de https://paginas.matem.unam.mx/matematicos/historia-del-instituto/facultad-deciencias-de-la-unam/departamento-de-matematicas-facultad-de-ciencias-unam/486breve-historia-de-las-matematicas-en-mexico 


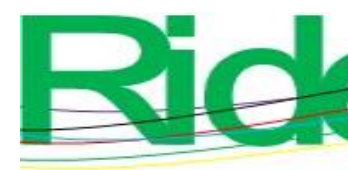

Revista Iberoamericana para la Investigación y el Desarrollo Educativo

ISSN 2007 - 7467

\begin{tabular}{|c|c|}
\hline Rol de Contribución & Autor (es) \\
\hline Conceptualización & $\begin{array}{l}\text { Dr. Claudio Rafael Vásquez Martínez (principal); Dr. Felipe } \\
\text { Anastacio González González (que apoya) }\end{array}$ \\
\hline Metodología & $\begin{array}{l}\text { Dr. Claudio Rafael Vásquez Martínez (principal); Dr. Felipe } \\
\text { Anastacio González González (que apoya). }\end{array}$ \\
\hline Software & $\begin{array}{l}\text { Dr. Claudio Rafael Vásquez Martínez (principal); Dr. Felipe } \\
\text { Anastacio González González (que apoya). }\end{array}$ \\
\hline Validación & $\begin{array}{l}\text { Dr. Claudio Rafael Vásquez Martínez (principal); Dr. Felipe } \\
\text { Anastacio González González (que apoya). }\end{array}$ \\
\hline Análisis Formal & $\begin{array}{l}\text { Dr. Claudio Rafael Vásquez Martínez (principal); Dr. Felipe } \\
\text { Anastacio González González (que apoya). }\end{array}$ \\
\hline Investigación & $\begin{array}{l}\text { Dr. Claudio Rafael Vásquez Martínez (principal); Dr. Felipe } \\
\text { Anastacio González González (que apoya). }\end{array}$ \\
\hline Recursos & $\begin{array}{l}\text { Dr. Claudio Rafael Vásquez Martínez (principal); Dr. Felipe } \\
\text { Anastacio González González (que apoya). }\end{array}$ \\
\hline Curación de datos & $\begin{array}{l}\text { Dr. Claudio Rafael Vásquez Martínez (principal); Dr. Felipe } \\
\text { Anastacio González González (que apoya). }\end{array}$ \\
\hline $\begin{array}{l}\text { Escritura - Preparación del } \\
\text { borrador original }\end{array}$ & $\begin{array}{l}\text { Dr. Claudio Rafael Vásquez Martínez (principal); Dr. Felipe } \\
\text { Anastacio González González (que apoya). }\end{array}$ \\
\hline $\begin{array}{l}\text { Escritura - Revisión y } \\
\text { edición }\end{array}$ & $\begin{array}{l}\text { Dr. Claudio Rafael Vásquez Martínez (principal); Dr. Felipe } \\
\text { Anastacio González González (que apoya). }\end{array}$ \\
\hline Visualización & $\begin{array}{l}\text { Dr. Claudio Rafael Vásquez Martínez (principal); Dr. Felipe } \\
\text { Anastacio González González (que apoya). }\end{array}$ \\
\hline Supervisión & $\begin{array}{l}\text { Dr. Claudio Rafael Vásquez Martínez (principal); Dr. Felipe } \\
\text { Anastacio González González (que apoya). }\end{array}$ \\
\hline $\begin{array}{l}\text { Administración de } \\
\text { Proyectos }\end{array}$ & $\begin{array}{l}\text { Dr. Claudio Rafael Vásquez Martínez (principal); Dr. Felipe } \\
\text { Anastacio González González (que apoya). }\end{array}$ \\
\hline Adquisición de fondos & $\begin{array}{l}\text { Dr. Claudio Rafael Vásquez Martínez (principal); Dr. Felipe } \\
\text { Anastacio González González (que apoya). }\end{array}$ \\
\hline
\end{tabular}

